\title{
Polynomial Harmonic Decompositions
}

\author{
Nicolae Anghel
}

\begin{abstract}
For real polynomials in two indeterminates a classical polynomial harmonic decomposition (cf. (1) below) is extended from square-norm divisors to conic ones. The main result is then applied to obtain a full polynomial harmonic decomposition, and to solve a Dirichlet problem with polynomial boundary data.
\end{abstract}

Harmonic functions are of utmost importance in analysis, geometry, and mathematical physics [1]. Even at their most basic occurrence, as polynomial harmonic functions, they produce surprisingly useful results. One of them is the following classical harmonic decomposition: In $\mathbf{R}^{n}, n \geq 2$, with coordinates $x=\left(x_{1}, x_{2}, \ldots, x_{n}\right)$, any homogeneous real polynomial of degree $m \geq 0$, $p_{m}(x)$, is uniquely decomposable as

$$
p_{m}(x)=h_{m}(x)+|x|^{2} p_{m-2}(x),
$$

where $h_{m}(x)$ is a homogeneous harmonic $\left(\sum_{j} \frac{\partial^{2} h_{m}}{\partial x_{j}^{2}}=0\right)$ real polynomial of degree $m,|x|^{2}=\sum_{j} x_{j}^{2}$, and $p_{m-2}(x)$ is a homogeneous polynomial of degree $m-2$ (possibly 0 ). As customary, here and in what follows the concepts of polynomial and polynomial function will be identified and used interchangeably.

There are vast generalizations of the decomposition (1), where the harmonic polynomials are replaced by polynomial solutions to constant coefficient partial differential operators, and $|x|^{2}$ by more general polynomials, like the symbols of those operators $[4,5]$. Key applications of the decomposition (1)

Key Words: Real polynomial, Harmonic function, Harmonic decomposition, Dirichlet problem.

2010 Mathematics Subject Classification: Primary 31A05; Secondary 31A25. 
are the determination of the spectrum of the Laplace operator on the Euclidean sphere $\mathbf{S}^{n-1}[6]$, or the simple (without a need for Poisson integrals) resolution of the Dirichlet problem with polynomial boundary data on the closed unit ball $B$ in $\mathbf{R}^{n}$ [2]. These applications and sheer curiosity made us wonder which hyperquadric functions

$$
\omega(x)=\sum_{j \leq k} c_{j k} x_{j} x_{k}+\sum_{j} c_{j} x_{j}+c, \quad c_{j k}, c_{j}, c \in \mathbf{R}
$$

could replace $|x|^{2}$ in the decomposition (1), of course at the unavoidable cost of losing homogeneity. In fact, in the language of [4] we will attempt to characterize all Fischer pairs of type $\left(\omega(x),|x|^{2}\right)$, with respect to the space of real polynomials in $\mathbf{R}^{n}$.

A superficial thought made us believe this would be the case precisely when $\Delta \omega:=\sum_{j} \frac{\partial^{2} \omega}{\partial x_{j}^{2}} \neq 0$, or equivalently $\sum_{j} c_{j j} \neq 0$. Furthermore, one way of possibly seeing this could be via an use of the classification theorem for hyperquadrics $\left(\left\{x \in \mathbf{R}^{n} \mid \omega(x)=0\right\}\right)$ up to rigid motions of $\mathbf{R}^{n}$, which leave the Laplace operator $\Delta$ invariant.

We were wrong on both accounts and while this is still work in progress we want to report here an answer in the case $n=2$, which we deem interesting enough to warrant this write-up. The main result of this note is the following

(Harmonic $\omega$-Decomposition) Theorem. Let $\omega(x)=a x_{1}^{2}+b x_{1} x_{2}+c x_{2}^{2}+$ $d x_{1}+e x_{2}+f, a, b, c, d, e, f \in \mathbf{R}, a^{2}+b^{2}+c^{2} \neq 0$ be a conic function on $\mathbf{R}^{2}$. The following two statements are equivalent:

(i) For any $m \geq 0$ and any real polynomial of degree $m$ in $x=\left(x_{1}, x_{2}\right)$, $p_{m}(x)$, there are unique real polynomials $h_{m}(x)$ and $p_{m-2}(x)$ of degrees respectively $m$ and $m-2, h_{m}(x)$ harmonic, i.e., $\Delta h_{m}=0$, such that

$$
p_{m}(x)=h_{m}(x)+\omega(x) p_{m-2}(x)
$$

(ii) Either $b^{2}-4 a c \leq 0$ or if $b^{2}-4 a c>0$ then $\frac{a+c+i \sqrt{b^{2}-4 a c}}{a+c-i \sqrt{b^{2}-4 a c}}$ is not $a$ complex root of unity.

For instance, according to the Theorem the unique decomposition (3) exists for $\omega(x)=x_{1}^{2}-x_{2}$, or for $\omega(x)=x_{1}^{2}+x_{2}^{2}+3 x_{1} x_{2}$, and it does not exist for $\omega(x)=x_{1}^{2}+x_{2}^{2}+4 x_{1} x_{2}$.

Couple of Lemmas, valid also in $\mathbf{R}^{n}$, will precede the proof of the Theorem.

Lemma 1. An unique decomposition of type (3) for polynomials on $\mathbf{R}^{n}$ exists, for $\omega(x)$ given by (2), if and only if there is no non-trivial harmonic polynomial of type $\omega(x) q(x)$, for $q(x)$ some (non-trivial) polynomial. 
Proof. The necessity is obvious, on the account of the uniqueness for the $\omega$ decomposition (3). For the sufficiency fix an integer $m \geq 0$ and let $p_{m}(x)$ be a real polynomial of degree $m$. If $\Delta p_{m}=0$ then the unique decomposition (3) holds by hypothesis, with $h_{m}(x)=p_{m}(x)$ and $p_{m-2}(x)=0$.

If $\Delta p_{m} \neq 0$, denote by $\mathcal{P}_{\leq(m-2)}$ the finite dimensional real vector space of real polynomials in indeterminates $x_{1}, x_{2}, \ldots, x_{n}$ of degree at most $m-2$. By hypothesis the linear map

$$
\mathcal{P}_{\leq(m-2)} \ni p \mapsto \Delta(\omega p) \in \mathcal{P}_{\leq(m-2)}
$$

is injective, and therefore surjective by a dimensionality argument. Thus, since $\Delta p_{m} \in \mathcal{P}_{\leq(m-2)}$ there is $p_{m-2} \in \mathcal{P}_{\leq(m-2)}$ such that $\Delta\left(\omega p_{m-2}\right)=\Delta p_{m}$, or equivalently $\Delta\left(p_{m}-\omega p_{m-2}\right)=0$. Setting $h_{m}(x):=p_{m}(x)-\omega(x) p_{m-2}(x)$, we have the existence of a decomposition of type (3). Its uniqueness follows again by hypothesis.

Lemma 2. An unique decomposition of type (3) for polynomials on $\mathbf{R}^{n}$ exists for $\omega(x)=\sum_{j \leq k} c_{j k} x_{j} x_{k}+\sum_{j} c_{j} x_{j}+c$ if and only if one exists for its homogeneous quadratic part $\tilde{\omega}(x)=\sum_{j \leq k} c_{j k} x_{j} x_{k}$.

Proof. Via the contrapositive of Lemma 1 it suffices to show that there is a non-trivial harmonic polynomial of type $\omega(x) q(x)$ if and only if there is one of type $\tilde{\omega}(x) \tilde{q}(x)$.

Assume first that $\omega(x) q(x)$ is a harmonic polynomial for some non-trivial polynomial $q(x)$ of degree $m \geq 0$. Then the homogeneous component of degree $m+2$ of $\omega(x) q(x)$ is $\tilde{\omega}(x) \tilde{q}(x)$, where $\tilde{q}(x)$ is the non-trivial homogeneous component of top degree $m$ of $q(x)$. Since $\Delta$ preserves homogeneity while lowering the degree by 2 it follows that $\Delta(\omega q)=0$ implies $\Delta(\tilde{\omega} \tilde{q})=0$, i.e., $\tilde{\omega}(x) \tilde{q}(x)$ is a non-trivial harmonic polynomial.

Conversely, assume now that $\tilde{\omega}(x) \tilde{q}(x)$ is a harmonic polynomial for some non-trivial polynomial $\tilde{q}(x)$. We can choose $\tilde{q}(x)$ such that it is homogeneous and its degree $m$ is the least possible. It follows that for any $0 \leq k \leq m-1$ the maps

$$
\mathcal{P}_{\leq k} \ni p \mapsto \Delta(\tilde{\omega} p) \in \mathcal{P}_{\leq k}
$$

are linear isomorphisms. We will provide a non-trivial polynomial $q(x)$ of degree $m$ such that $\Delta(\omega q)=0$ in the following way: In the homogeneous decomposition of $q(x), q(x)=\sum_{j=0}^{m} q_{m-j}(x), q_{m-j}(x)$ homogeneous polynomial of degree $m-j$, we set first $q_{m}(x):=\tilde{q}(x)$. Write now $\omega(x)=$ $\omega_{2}(x)+\omega_{1}(x)+\omega_{0}(x)$, where $\omega_{2}(x):=\tilde{\omega}(x), \omega_{1}(x):=\sum_{j} c_{j} x_{j}$, and $\omega_{0}(x):=c$. Then the homogeneous decomposition of $\omega(x) q(x)$ is

$$
\omega q=\omega_{2} q_{m}+\left(\omega_{2} q_{m-1}+\omega_{1} q_{m}\right)+\left(\omega_{2} q_{m-2}+\omega_{1} q_{m-1}+\omega_{0} q_{m}\right)+\cdots+\omega_{0} q_{0},
$$


while the homogeneous decomposition of $\Delta(\omega q)$ is

$$
\Delta(\omega q)=\Delta\left(\omega_{2} q_{m-1}+\omega_{1} q_{m}\right)+\Delta\left(\omega_{2} q_{m-2}+\omega_{1} q_{m-1}+\omega_{0} q_{m}\right)+\cdots .
$$

The linear isomorphisms (4) yield now recursively the homogeneous polynomials $q_{m-1}(x), q_{m-2}(x), \ldots, q_{0}(x)$, such that $\Delta\left(\omega_{2} q_{m-1}\right)=-\Delta\left(\omega_{1} q_{m}\right)$, $\Delta\left(\omega_{2} q_{m-2}\right)=-\Delta\left(\omega_{1} q_{m-1}+\omega_{0} q_{m}\right), \ldots$, making the right-hand-side of $(5)$ vanish.

In preparation for proving the Harmonic $\omega$-Decomposition Theorem we specialize to the case $n=2$ and extend the polynomial ring $\mathbf{R}\left[x_{1}, x_{2}\right]$ to its complexification $\mathbf{C}\left[x_{1}, x_{2}\right]$. There is an obvious conjugation operator $\mathbf{C}\left[x_{1}, x_{2}\right]$ $\ni p \mapsto \bar{p} \in \mathbf{C}\left[x_{1}, x_{2}\right]$ and then $p \in \mathbf{C}\left[x_{1}, x_{2}\right]$ belongs to $\mathbf{R}\left[x_{1}, x_{2}\right]$ if and only if $p=\bar{p}$. Notice that $\mathbf{C}\left[x_{1}, x_{2}\right]=\mathbf{C}[z, \bar{z}]$, where $z=x_{1}+i x_{2}$ and $\bar{z}=x_{1}-i x_{2}$, $i=\sqrt{-1}$. A homogeneous polynomial of degree $m$ in $\mathbf{C}\left[x_{1}, x_{2}\right]$ is therefore uniquely representable as

$$
p(z, \bar{z})=\sum_{j=0}^{m} \gamma_{j} z^{m-j} \bar{z}^{j}, \quad \gamma_{j} \in \mathbf{C},
$$

and it belongs to $\mathbf{R}\left[x_{1}, x_{2}\right]$ if and only if $\alpha_{m-j}=\bar{\alpha}_{j}$ for every $j$.

As an operator from $\mathbf{R}\left[x_{1}, x_{2}\right]$ into itself $\Delta=\frac{\partial^{2}}{\partial x_{1}^{2}}+\frac{\partial^{2}}{\partial x_{2}^{2}}$ extends by complex linearity to $\mathbf{C}[z, \bar{z}]$ according to the formula $\Delta=4 \frac{\partial^{2}}{\partial z \partial \bar{z}}$, where $\frac{\partial}{\partial z}=\frac{1}{2}\left(\frac{\partial}{\partial x_{1}}+\frac{1}{i} \frac{\partial}{\partial x_{2}}\right)$ and $\frac{\partial}{\partial \bar{z}}=\frac{1}{2}\left(\frac{\partial}{\partial x_{1}}-\frac{1}{i} \frac{\partial}{\partial x_{2}}\right)$. Consequently,

$$
\Delta\left(\sum_{j=0}^{m} \gamma_{j} z^{m-j} \bar{z}^{j}\right)=4 \sum_{j=1}^{m-1} j(m-j) \gamma_{j} z^{m-j-1} \bar{z}^{j-1}
$$

A polynomial $p(z, \bar{z}) \in \mathbf{C}[z, \bar{z}]$ will be called harmonic if $\frac{\partial^{2} p}{\partial z \partial \bar{z}}=0$. A harmonic polynomial $h\left(x_{1}, x_{2}\right) \in \mathbf{R}\left[x_{1}, x_{2}\right]$ is also harmonic in $\mathbf{C}[z, \bar{z}]$, and conversely if $g(z, \bar{z})$ is harmonic then $\operatorname{Re} g:=\frac{g+\bar{g}}{2}$ and $\operatorname{Im} g:=\frac{g-\bar{g}}{2 i}$ are harmonic in $\mathbf{R}\left[x_{1}, x_{2}\right]$.

Proof of the Harmonic $\omega$-Decomposition Theorem. For $x=\left(x_{1}, x_{2}\right) \in \mathbf{R}^{2}$ let $\tilde{\omega}(x):=a x_{1}^{2}+b x_{1} x_{2}+c x_{2}^{2} \in \mathbf{R}\left[x_{1}, x_{2}\right]$, where $a, b, c \in \mathbf{R}, a^{2}+b^{2}+c^{2} \neq 0$. The representation of $\tilde{\omega}(x)$ in $\mathbf{C}[z, \bar{z}]$ is then

$$
\tilde{\omega}(x)=\tilde{\omega}(z, \bar{z})=\frac{1}{4}\left(\alpha z^{2}+\beta z \bar{z}+\overline{\alpha z}^{2}\right),
$$

where $\alpha=(a-c)-b i$ and $\beta=2(a+c)$. In view of the two lemmas and the above considerations it suffices to show that there is some non-trivial 
homogeneous complex polynomial $p(z, \bar{z}) \in \mathbf{C}[z, \bar{z}]$ such that $\tilde{\omega}(z, \bar{z}) p(z, \bar{z})$ is harmonic if and only if $b^{2}-4 a c>0$ and $\frac{a+c+i \sqrt{b^{2}-4 a c}}{a+c-i \sqrt{b^{2}-4 a c}}$ is a complex root of unity.

Writing $p(z, \bar{z})$ as in $(6)$, for some non-negative integer $m$, we see that

$$
\begin{aligned}
& \tilde{\omega}(z, \bar{z}) p(z, \bar{z}) \\
& =\frac{1}{4}\left(\sum_{j=0}^{m} \alpha \gamma_{j} z^{m-j+2} \bar{z}^{j}+\sum_{j=0}^{m} \beta \gamma_{j} z^{m-j+1} \bar{z}^{j+1}+\sum_{j=0}^{m} \bar{\alpha} \gamma_{j} z^{m-j} \bar{z}^{j+2}\right),
\end{aligned}
$$

and then by (7)

$$
\begin{aligned}
& \Delta(\tilde{\omega} p)(z, \bar{z}) \\
& =\sum_{j=1}^{m} j(m-j+2) \alpha \gamma_{j} z^{m-j+1} \bar{z}^{j-1}+\sum_{j=0}^{m}(j+1)(m-j+1) \beta \gamma_{j} z^{m-j} \bar{z}^{j} \\
& +\sum_{j=0}^{m-1}(j+2)(m-j) \bar{\alpha} \gamma_{j} z^{m-j-1} \bar{z}^{j+1}=(m+1)\left(\alpha \gamma_{1}+\beta \gamma_{0}\right) z^{m} \\
& +\sum_{j=1}^{m-1}(j+1)(m-j+1)\left(\alpha \gamma_{j+1}+\beta \gamma_{j}+\bar{\alpha} \gamma_{j-1}\right) z^{m-j} \bar{z}^{j} \\
& +(m+1)\left(\beta \gamma_{m}+\bar{\alpha} \gamma_{m-1}\right) \bar{z}^{m} .
\end{aligned}
$$

Therefore, $\Delta(\tilde{\omega} p)=0$ if and only if

$$
\left\{\begin{aligned}
\alpha \gamma_{1}+\beta \gamma_{0} & =0 \\
\alpha \gamma_{j+1}+\beta \gamma_{j}+\bar{\alpha} \gamma_{j-1} & =0, \quad j=1,2, \ldots, m-1 \\
\beta \gamma_{m}+\bar{\alpha} \gamma_{m-1} & =0
\end{aligned}\right.
$$

Setting

$$
\gamma_{-1}:=0 \text { and } \gamma_{m+1}:=0,
$$

(9) can be written in the more compact form

$$
\alpha \gamma_{j+1}+\beta \gamma_{j}+\bar{\alpha} \gamma_{j-1}=0, \quad j=0,1, \ldots, m-1, m .
$$

We are interested in necessary and sufficient conditions under which the system (11) admits non-trivial solutions $\gamma_{j}$, subject to the constraints (10). Clearly, for this to happen it is necessary that $\alpha \neq 0$, in which case (11) becomes a 
constrained 2 nd order linear recurrence. Its characteristic equation $\alpha r^{2}+\beta r+$ $\bar{\alpha}=0$ has complex solutions

$$
r_{1,2}=\frac{-\beta \pm \sqrt{\beta^{2}-4 \alpha \bar{\alpha}}}{2 \alpha}=\frac{-(a+c) \pm \sqrt{4 a c-b^{2}}}{(a-c)-i b} .
$$

Notice that $r_{1,2}$ cannot vanish. As customary, any unconstrained solution of (11) depends on two complex constants $A$ and $B$ and is given by

$$
\gamma_{j}=\left\{\begin{array}{ll}
A r_{1}^{j}+B r_{2}^{j}, & \text { if } 4 a c-b^{2} \neq 0 \\
A r_{1}^{j}+B j r_{1}^{j}, & \text { if } 4 a c-b^{2}=0
\end{array} \quad j=-1,0,1, \ldots, m, m+1 .\right.
$$

If $4 a c-b^{2} \neq 0$ then $\gamma_{-1}=0$ gives $B=-A \frac{r_{2}}{r_{1}}$, so non-trivial solutions require $A \neq 0 . \quad \gamma_{m+1}=0$ then yields $A\left(\frac{r_{1}^{m+2}-r_{2}^{m+2}}{r_{1}}\right)=0$, or equivalently $\left(\frac{r_{1}}{r_{2}}\right)^{m+2}=1$. By (12) this is possible precisely when $b^{2}-4 a c>0$ and $\frac{a+c+i \sqrt{b^{2}-4 a c}}{a+c-i \sqrt{b^{2}-4 a c}}$ is a complex $(m+2)$-root of unity.

If $b^{2}-4 a c=0$ then $\gamma_{-1}=0$ implies $A=B$ and so $\gamma_{m+1}=\frac{(m+2) A}{r_{1}^{m+1}}$ vanishes only if $A=0$. Consequently, there are no non-trivial solutions in this case. The proof of the Theorem is complete.

Of course, it would be of great interest to have an algorithmic way of generating the harmonic part $h_{m}$ of $p_{m}$ in the $\omega$-Decomposition (3) without solving linear systems, just like the one found in [2] for the classical case $\omega(x)=$ $|x|^{2}$. However, such an algorithm is highly unlikely to exist for arbitrary $\omega$.

We conclude this note with two applications of the Theorem: the first one is a standard full harmonic $\omega$-decomposition of real polynomials in two variables, and the second one is a solution to Dirichlet problems on elliptic or parabolic regions in $\mathbf{R}^{2}$, with polynomial boundary data.

(Full Harmonic $\omega$-Decomposition) Theorem. Let $a, b, c, d, e, f \in \mathbf{R}, a^{2}+$ $b^{2}+c^{2} \neq 0$ be such that either $b^{2}-4 a c \leq 0$ or if $b^{2}-4 a c>0$ then $\frac{a+c+i \sqrt{b^{2}-4 a c}}{a+c-i \sqrt{b^{2}-4 a c}}$ is not a complex root of unity. Then for any integer $m \geq 0$ and for any real polynomial of degree $m$ in $x=\left(x_{1}, x_{2}\right), p_{m}(x)$, there are unique harmonic polynomials $h_{m}(x), h_{m-2}(x), \ldots, h_{m-2 k}(x)$, where $k=\left[\frac{m}{2}\right]$, such that

$$
p_{m}(x)=h_{m}(x)+\omega(x) h_{m-2}(x)+\omega^{2}(x) h_{m-4}(x)+\cdots+\omega^{k}(x) h_{m-2 k}(x),
$$


and where as above $\omega(x)=a x_{1}^{2}+b x_{1} x_{2}+c x_{2}^{2}+d x_{1}+e x_{2}+f$.

Proof. The Full Decomposition Theorem is obvious for $m=0$ or 1 , since then $p_{m}(x)$ is harmonic. For $m \geq 2$ it can be proved by induction on $m$, the inductive step clearly being implementable by the $\omega$-Decomposition (3). The uniqueness of the harmonic polynomials in (14) follows from the uniqueness of $h_{m}(x)$ and $p_{m-2}(x)$ in (3).

Recall now the Dirichlet problem in $\mathbf{R}^{n}$ : given a non-empty open subset $\Omega \subset \mathbf{R}^{n}$, for any real-valued continuous function $\phi$ on its boundary $\partial \Omega$, find (an eventually unique) continuous function $h$ on $\bar{\Omega}$, such that $h$ is harmonic (class $C^{2}$, and satisfies $\Delta h=0$ ) on $\Omega$ and restricts to $\phi$ on $\partial \Omega$. For certain (possibly degenerate) conic regions in $\mathbf{R}^{2}$ with polynomial boundary data we have the following

(Polynomial Dirichlet Problem) Theorem. Let $\omega(x)=a x_{1}^{2}+b x_{1} x_{2}+$ $c x_{2}^{2}+d x_{1}+e x_{2}+f, a, b, c, d, e, f \in \mathbf{R}, a^{2}+b^{2}+c^{2} \neq 0$ be a conic function on $\mathbf{R}^{2}$ be such that either $b^{2}-4 a c \leq 0$ or if $b^{2}-4 a c>0$ then $\frac{a+c+i \sqrt{b^{2}-4 a c}}{a+c-i \sqrt{b^{2}-4 a c}}$ is not a complex root of unity, and such that $\Omega:=\left\{x \in \mathbf{R}^{2} \mid \omega(x)<0\right\}$ is nonempty. Then for any polynomial $p \in \mathbf{R}\left[x_{1}, x_{2}\right]$ there is a polynomial solution to the Dirichlet problem on $\bar{\Omega}=\left\{x \in \mathbf{R}^{2} \mid \omega(x) \leq 0\right\}$ which takes the value $p$ on $\partial \Omega=\left\{x \in \mathbf{R}^{2} \mid \omega(x)=0\right\}$. The solution to this polynomial Dirichlet problem may not be unique, however it is so if $\Omega$ is bounded.

Proof. The existence of a polynomial solution to this Dirichlet problem is pretty simple, in view of the Harmonic $\omega$-Decomposition Theorem. Indeed, we have by (3), $p(x)=h(x)+\omega(x) q(x)$, with $h(x)$ a harmonic polynomial of the same degree as $p(x)$, and $q(x)$ some other polynomial. Since $h(x)=p(x)$ when $\omega(x)=0, h$ is such a desired solution.

To see that the solution is not unique in general, let $\omega(x)=-x_{2}^{2}$. This $\omega$ satisfies the hypotheses of the Theorem on $\Omega=\left\{x \in \mathbf{R}^{2} \mid x_{2} \neq 0\right\}$ and $h_{1}(x)=0$ and $h_{2}(x)=x_{2}$ are two distinct harmonic functions on $\bar{\Omega}=\mathbf{R}^{2}$ which restrict to the same value, 0 , on $\partial \Omega=\left\{x \in \mathbf{R}^{2} \mid x_{2}=0\right\}$.

However, when $\Omega$ is bounded, or equivalently $\partial \Omega$ is an ellipse and $\Omega$ its interior, if $\tilde{h}$ is another solution to the Dirichlet problem with boundary value $p$, then $\tilde{h}-h$ is a harmonic function vanishing on $\partial \Omega$, and by the maximum/minimum principle for harmonic functions [3], $\tilde{h}=h$ on $\bar{\Omega}$.

\section{References}

[1] S. Axler, P. Bourdon, W. Ramey, Harmonic Function Theory, Graduate Texts Math. 137, Springer, New-York, 1992. 
[2] S. Axler, W. Ramey, Harmonic Polynomials and Dirichlet-Type Problems, Proc. Amer. Math. Soc. 123 (1995) 3765-3773.

[3] W. Conley, Complex Analysis, Birkhäuser, Boston, 2004.

[4] F. Colombo, I. Sabadini, F. Sommen and D. Struppa, Analysis of Dirac Systems and Computational Algebra, Birkhäuser, Boston, 2004.

[5] B. Reznick, Homogeneous Polynomial Solutions to Constant Coefficient PDE's, Adv. Math. 117 (1996) 179-192.

[6] M. Shubin, Pseudodifferential Operators and Spectral Theory, Springer, NewYork, 1987.

Nicolae ANGHEL,

Department of Mathematics,

University of North Texas,

Denton, TX 76203, USA.

Email: anghel@unt.edu 